\title{
Duration of Untreated Psychosis and Cognitive Functioning
}

Charlotte Rapp, MSc; Erich Studerus, PhD; Hilal Bugra, MSc; Jacqueline Aston, MD;

Corinne Tamagni, PhD; Anna Walter, MD; Stefan Borgwardt, MD; Anita Riecher-

Rössler, MD*

University Psychiatric Outpatient Department, University of Basel Psychiatric Clinics, c/o University Hospital Basel, Petersgraben 4, CH-4031 Basel, Switzerland

*Corresponding author:

Prof. A. Riecher-Rössler

University of Basel Psychiatric Clinics

Center for Gender Research and Early Detection

c/o University Hospital Basel

Petersgraben 4

$\mathrm{CH}-4031$ Basel

Switzerland

Phone: +41 612655114

Fax: +41 612654599

E-mail: Anita.Riecher@upkbs.ch 


\section{Abstract}

Background: Studies examining the influence of duration of untreated psychosis (DUP) or duration of untreated illness (DUI) on cognition vary with regards to results and methods. This study is the first in this field to include an at risk mental state with later transition to psychosis (ARMS-T) sample and to analyse how the DUI relates to their cognitive functioning. Because methodological operationalization of cognitive functioning in previous studies is highly heterogeneous, we aimed to compare different approaches.

Method: 60 FEP and 24 ARMS-T patients were examined. Associations between DUP, DUI and neurocognitive performance were tested by three different operationalisations of cognition: as the raw outcome measure of different neuropsychological tests, as outcome scores which were normed on a sample of 75 healthy participants, and as the deterioration index (DI).

Results: There were no significant correlations between DUP or DUI and outcome of neuropsychological tests in both normed and raw scores. When adjusted for covariates, DUP and DUI also did not significantly predict any cognitive performance. There was no significant relationship between DUP or DUI and the DI index. However, longer DUP and DUI were significantly associated with stronger negative symptoms.

Conclusions: This study could not confirm an association between duration of untreated psychosis or duration of untreated illness and neurocognitive performance in an ARMS and FEP sample. This could be because schizophrenic psychoses are neurodevelopmental disorders in which most cognitive deficits exist long before the onset of psychiatric symptoms.

Keywords: First episode psychosis (FEP), at risk mental state (ARMS), neuropsychology, cognitive deterioration, duration of untreated illness, duration of untreated psychosis 


\section{Introduction}

Duration of untreated psychosis (DUP) is defined as the time from appearance of the first psychotic symptom to initiation of adequate neuroleptic treatment (Marshall et al., 2005). Shorter DUP is associated with better clinical outcome (Marshall et al., 2005) and greater response to antipsychotic treatment (Perkins et al., 2005).

The specific association between DUP and cognitive deficits at treatment initiation has been analysed in several studies and gained importance based on the hypothesis that psychosis might have a "toxic effect" on the brain (Wyatt, 1991). However, among 18 studies which have so far examined this association, only 6 found a positive association whereas 13 did not (supplementary Table 1). The studies vary with regard to methods and operationalization of DUP, which makes these data difficult to interpret. Some of these studies also considered the relationship of cognitive deficits with duration of untreated illness (DUI), which is usually defined as the DUP plus any period of prodromal symptoms (e.g. Barnes et al., 2000).

Most of the existing studies analysed the association between DUP and cognitive functioning by relating DUP to different neuropsychological tests and IQ measures at the time of treatment initiation (e.g. Goldberg et al., 2009). However, Amminger et al. (2002b) argued that only the difference to a patient's premorbid abilities would provide a meaningful measure for deterioration. Consequently, they made use of the Deterioration Index (DI; Bilder et al., 1985), assessing the discrepancy between "hold" and "non-hold" cognitive function. In accordance with the above hypothesis, Amminger et al. (2002a) found that DUP was positively associated with DI, but not with morbid cognitive functioning, which was replicated by Gaynor et al. (2009).

Because methodological operationalization of cognitive functioning in studies examining the relationship between DUP and cognitive decline is highly heterogeneous, we aimed to compare different approaches in order to detect whether differences in methodological approaches could have led to inconsistencies between earlier studies. Specifically, we analysed 
the relationship between DUP, DUI and cognitive deterioration in three different ways; first, we operationalized cognitive functioning as raw outcome measure of different neuropsychological tests as done in most similar studies. Second, we analysed the relationship between DUP, DUI, and cognitive deficits using neuropsychological outcome scores normed on a sample of 75 healthy participants with the same socio-demographic characteristics. Thirdly, deterioration was assessed by the DI in a similar way as in Amminger et al. (2002a) and Gaynor et al. (2009).

Of the studies listed in supplementary Table 1, only four have also looked at the interrelationship between DUI (other than DUP) and cognition. None of them found significant correlations. A difference between the effect of DUP versus DUI on cognitive performance might be expected in the way that the effect of DUP could be stronger than the effect of DUI on cognition as the active brain disturbance causing toxicity to neuronal growth and repair might be more distinct due to acute positive psychotic symptoms (relating to DUP) than unspecific symptoms such as depression or concentration difficulties (relating to DUI).

This study is the first to include 24 at risk mental state (ARMS) individuals with later transition to psychosis (ARMS-T) and to examine how the DUI relates to their cognitive functioning. The inclusion of an ARMS-T sample can give important indications as to the course of the influence of first psychiatric symptoms on cognition, eventually detecting that first psychiatric symptoms already influence cognition before the outbreak of psychosis. However, based on previous studies with first-episode patients reporting no relationship between DUI and cognition (e.g. Barnes et al., 2000; Norman and Malla, 2001), we expected no relationship between DUI and cognitive functioning of ARMS-T patients, which would underline the assumption that cognitive deficits occur prior to the onset of psychiatric symptoms. 


\section{Methods}

\subsection{Setting and recruitment}

This study was part of the Basel FePsy study (Früherkennung von Psychosen) (RiecherRössler et al., 2007a; Riecher-Rössler et al., 2009), which aims to improve the early detection of psychosis. Participants were recruited into the study via a specialised early detection outpatient clinic at the Psychiatric Outpatient Department Basel. The study was approved by the Ethics Committee of Basel, Switzerland (EKBB), and written informed consent was obtained from the participants.

\subsection{Screening procedure}

The Basel Screening Instrument for Psychosis (BSIP) was used (Riecher-Rössler et al., 2008) to assess participants and identify them as individuals with an at risk mental state (ARMS), first episode psychosis (FEP) patients, or "not at risk for psychosis" (i. e. other psychiatric diseases). Inclusion as ARMS required one or more of the following: (a) "attenuated" psychotic symptoms, (b) brief limited intermittent psychotic symptoms (BLIPS), (c) a first degree relative with a psychotic disorder plus at least two indicators of a clinical change, such as marked decline in social or occupational functioning, or (d) minimal account and combination of unspecific risk factors according to the BSIP (Riecher-Rossler et al., 2008). Assignment to the FEP group was based on the transition criteria described by Yung et al (Yung et al., 1998). Subjects treated with antipsychotics for $>3$ weeks or with a clearly diagnosed organic, substance induced or affective psychosis were excluded.

\subsection{Participants}

In this study, we present data of 84 patients from the Basel FePsy study for which information about DUP, DUI, and neuropsychological performance could be obtained. 28 patients from the FePsy study had to be excluded from this study due to missing data regarding 
DUP, DUI or neuropsychology. 60 of the patients were identified as FEP patients. 24 ARMS individuals were included, who later made the transition to frank psychosis (ARMS-T) according to the PACE criteria (Yung et al., 1998). This sample overlaps with previous samples reported on from the FePsy study (Pflueger et al., 2007; Riecher-Rössler et al., 2009).

A sample of 75 healthy participants was used for the normalisation of the neuropsychological tests. They were recruited from a commercial school, hospital staff, and through advertisements. Exclusion criteria were: a current or former psychiatric disorder or neurological disease, serious medical condition, substance abuse, or a family history of psychiatric disorder.

\subsection{Duration of untreated illness/duration of untreated psychosis}

The duration of untreated illness (DUI) was defined as the time period between first selfperceived signs or symptoms of a change in well-being and first contact with our early detection service. The DUI was determined by using the Basel Interview for Psychosis (BIP) (RiecherRössler et al., 2007a), a structured and specifically developed interview for the early detection of psychosis, and was assessed both for ARMS-T and FEP patients at first contact.

The duration of untreated psychosis (DUP) was defined as time period between the appearance of the first positive psychotic symptom and first contact with our early detection service. The DUP was only assessed in FEP patients as it was almost zero in our ARMS-T patients due to our close follow-up during the at-risk-mental-state and prompt treatment at transition. Both DUP and DUI were established by considering the patients subjective response but also including other clinical information resources such as for instance information from family members. 


\subsection{Neurocognitive measures}

The neuropsychological test-battery was mainly based on computer-administered tests, so that nearly all measures provided reaction times and numbers of errors (omissions/false alarms). The assessment of the participants was conducted by fully qualified psychologists and well-trained, supervised advanced students of psychology and was conducted at time of first contact with our service for both ARMS and FEP.

Intelligence

- The Mehrfachwahl-Wortschatz-Test (MWT-A; Lehrl, 1991) and the Leistungsprüfsystem, scale 3 (LPS) (Horn, 1983), are well established German intelligence scales for assessing verbal and non-verbal (abstract reasoning) abilities.

Executive function

- The computer-administered Tower of Hanoi (ToH; Gediga, 1994) is a task demanding sequential anticipation of the consequences of one's actions. The execution of action needs to be guided by planned and goal-oriented behaviour.

- The computer-administered Wisconsin Card Sorting Test (WCST; Drühe-Wienholt, 1998; Heaton, 1993) demands flexible shifts between three cognitive sets in order to avoid perseveration errors. The execution of action is controlled by a task-related feedback.

- The Go/No-Go subtest of the Tests for Attentional Performance (TAP; Zimmermann, 1993) requires the inhibition of responses provoked by visually similar, but non-target stimuli. Working memory

- The Working Memory subtest of the Tests for Attentional Performance (TAP; Zimmermann, 1993) forces the subject to match visually presented stimuli in terms of a 2-back task.

Whether the subject succeeds depends on the subject's ability to concentrate on tasks which impose a permanent cognitive load.

\section{Attention}


- The Continuous Performance Test (CPT-OX; Rosvold et al., 1956) measures vigilance in terms of sustained visual attention. Four letters are consecutively displayed in a pseudorandomized order. Whenever a prime $(\mathrm{O})$ precedes the target $(\mathrm{X})$ a pushbutton needs to be pressed.

It permits the computation of indices which reflect performance changes between the first and the second part of the task. CPT-OX version is therefore equivalent to CPT-AX.

\subsection{DI index}

The DI index (Bilder et al., 1992) bases on the assumption that a large discrepancy between a subject's best and poorest cognition performance suggests cognitive loss. The DI index by Bilder et al. (1992) is calculated by assessing the difference between the Wechsler Adult Intelligence Scale-R (Wechsler, 1981) subtests: Information and Vocabulary ("hold-tests") to the Digit symbol ("non-hold" test) according the following formula: "hold-tests" - "non-hold tests" / "hold-tests". In our study, we used the MWT-A (Lehrl, 1991) test as "hold-test", which has been shown to be a useful measure of premorbid IQ (Lehrl et al., 1995). The LPS, scale 3 (Horn, 1983) which is a german intelligence scale for assessing non-verbal abstract reasoning abilities, was used as "non-hold" test. Consequently, the DI index in our study was computed as follows:

$$
D I=\frac{(\text { MWT-A })-(\text { LPS })}{(\text { MWT-A })}
$$

\subsection{Psychopathology measures}

Positive symptoms were assessed with the 4 items: hallucinations, suspiciousness, unusual thought content, and conceptual disorganisation of the Brief Psychiatric Rating Scale (BPRS) (Ventura et al., 1993). Negative symptoms were assessed with the Scale for the Assessment of Negative Symptoms (SANS) (Andreasen, 1983). 


\subsection{Cannabis use}

Cannabis use was determined by using the Basel Interview for Psychosis (BIP) (RiecherRössler et al., 2007b) and was assessed both for ARMS and FEP patients at study inclusion. The BIP contains two items assessing the frequency of past and present cannabis consumption. Frequency of cannabis use is assessed by these items on a five-point ordinal scale using the following response categories: daily, several times a week, several times a month, less than several times a month, and not at all. For the present analyses, only current cannabis use was considered.

\subsection{Statistical analysis}

All analyses were performed using SPSS version 19. Associations between DUP, DUI and neurocognitive performance were tested with and without adjusting for confounding variables and with three different operationalizations of neurocognitive performance. Due to a strong positive skew in both DUP and DUI, Spearman's rank correlation was used in the unadjusted analyses and log transformed DUP/DUI in the adjusted analyses (i.e. multiple regressions). Adjustments were performed for the influence of age, gender, years of education, antipsychotic medication, cannabis use, BPRS positive symptom score, and SANS total score.

For the first operationalization of neurocognitive performance, we calculated composite scores for each subtest of the neuropsychological test battery. The composites were the averages of the z-transformed performance scores (i.e. reaction-time, omissions, false-alarms) of each test. For the second operationalization, we used composite scores that were the averages of the normed performance scores of each test. Scores were normed on our sample of 75 healthy participants according the following procedure: first, the distribution of the residuals of each score in the normative sample was made approximately Gaussian by applying the Box-Cox transformation for linear models (Box and Cox, 1964) if the score was uncensored and the Box- 
Cox transformation for Tobit models (Han and Kronmal, 2004) if the score was censored. Second, an optimal model was selected among six possible models with the following predictor sets: intercept only, age, gender, education, age+education, age+sex, age+education+sex, age +education+sex+age-sex-interaction. Again, Tobit and linear regression models were used for censored and uncensored scores, respectively. Optimal models were selected by using the predicted residual sum-of-squares (PRESS) statistic (Allen, 1974), which measures the predictive performance of a model in leave-out-cross-validation. Finally, normed values were calculated according to the following formula: (transformed raw score - predicted from best model)/residual standard error of best model.

For the third operationalization of neurocognitive decline, a DI index was calculated as described above.

P-values $<0.05$ were considered significant. However, for hypothesis tests using the first and second operationalization of neurocognitive decline, which comprised seven different outcome measures, we divided the alpha level by seven to account for multiple testing.

\section{Results}

\subsection{Sample characteristics and confounding factors}

The patients included in this study did not differ from those who were excluded regarding to age, gender, education, total SANS, BPRS positive symptom and BPRS total score (Table 1).. There were also no significant differences between the ARMS-T and the FEP group included in the study regarding age $(p=0.05)$, gender $(p=0.80)$, education $(p=0.59)$, cannabis use $(p=0.77)$ and SANS total score $(p=0.69)$. However, FEP patients had higher BPRS positive symptoms score $(p<0.001)$ and were significantly more likely to be medicated with antipsychotics $(p=0.04)$. $3(12.5 \%)$ ARMS-T and 23 (38.3\%) FEP subjects had been receiving neuroleptic treatment at assessment time. 
Insert Table 1 here

\subsection{Duration of untreated illness and duration of untreated psychosis}

The median DUI was 36 months (mean: 56.7 months, SD: 68.67 months, IQR: 75.0). DUI did not differ significantly between FEP and ARMS-T (Mann-Whitney-U: 741; $p=0.839$ ), nor between men and women (Mann-Whitney-U: 725.5; $p=0.581$ ). The median DUP, which by definition could only be assessed in FEP patients, was 12 months with a mean of 36.8 months (SD: 56.43, IQR: 53.75) months. The large difference between mean and median DUP was due to a positively skewed distribution (skewness: 2.70 , kurtosis: 8.39 ), caused by a small number of outliers. Regarding gender differences, the median value of DUP for men was 12 months (mean: 40.8 months; SD: 62.67 ; IQR: 56.75), while the median value of DUP for women was 11 months (mean: 28.8 months; SD: 41.55; IQR: 46.00), which was statistically non-significant (MannWhitney-U: 368; $p=0.62)$. Figure 1 shows the DUP and DUI distributions in the different subgroups.

Insert Figure 1 here

There were no significant correlations between DUP or DUI, cannabis use, years of education and BPRS total score. There was a significant correlation between DUP and SANS global score $(r=0.23, p=0.024)$ as well as a significant correlation between DUI and SANS global score $(r=0.20, p=0.034)$.

\subsection{DUP, DUI and neuropsychological tests}

Table 2 lists Spearman's rank correlations of DUP and DUI with the normed composite scores and composite scores based on z-transformed scores of the neuropsychological tests for the whole group of FEP and ARMS-T patients. 
Insert Table 2 here

There were no significant correlations between DUI or DUP and normed composite scores or composite scores based on z-transformed scores of neuropsychological tests, even when no adjustment for multiple testing was applied (Table 2). The results did not change when the analysis was performed separately for ARMS-T and FEP (Table 3).

Insert Table 3 here

In the confounder-adjusted analyses (i.e. multiple regression models) with normed composite scores, DUP was only associated with one neurocognitive outcome variable, namely, non-verbal intelligence as measured by the LPS $(p=0.03)$, but the association was relatively weak $(\beta=0.28)$, not in the expected direction, and did not remain significant after correction for multiple testing $(p=0.15)$. The same was true for DUI, which showed one positive association with LPS $(\beta=0.23 ; p=0.03$ ) that did not survive correction for multiple testing (adjusted $p=0.22$ ). As can be seen from Figure 2, only age, years of education and SANS total score were significantly associated with any outcome when adjusted for potential confounders and multiple testing. The results of the confounder-adjusted analyses did not change when repeated with composite scores based on z-transformed scores.

Insert Figure 2 here

\subsection{DUP, DUI and the Deterioration Index}

The median value for the deterioration index (DI) was 0.01 (mean: -0.01 ; SD: 0.17 ). DI scores did not differ between ARMS-T and FEP ( $T=-1.09 ; p=0.28)$ and between women and men $(T=1.74 ; p=0.09)$. There was a significant correlation between $D I$ and age $(r=0.31 ; p=$ 
$0.004)$ as well as DI and BPRS score ( $r=0.24 ; p=0.021)$, but no significant correlations between DI and DUI, DUP, SANS total score, gender, years of education, and cannabis use. In a multiple regression model, where DI was entered as the dependent variable and DUP, BPRS positive symptom score, SANS total score, gender, years of education, cannabis use and antipsychotic medication as predictors, only age predicted a stronger cognitive decline (standardised regression coefficient $\beta=0.271 ; p=0.019$ ).

\section{Discussion}

The aim of this study was to investigate the relationship between DUP, DUI and neurocognitive performance in a sample of 60 FEP and 24 ARMS patients with later transition to psychosis (ARMS-T). We compared three ways of operationalizing cognitive functioning but neither of the approaches detected a relationship between cognitive deficits and DUP/DUI.

\subsection{Length of DUP or DUI and influencing factors}

The mean and median DUP of our study (148 weeks/48 weeks) were somewhat higher than reported in most other studies (for review Norman and Malla, 2001). This might be explained by the way our onset of DUP was set; DUP started when the first positive psychotic symptom ever occurred, regardless of severity and frequency of the symptom. Therefore, our DUP already started when the first "attenuated" or "brief-limited-intermittent" psychotic symptoms were subjectively experienced. But even if it is true that a prolonged DUP/DUI might be associated with cognitive deterioration, this deterioration itself might have consequences in terms of the accuracy of information obtained from the patient and have contributed to a recall bias. However, the fact that we also integrated other clinical information in order to establish DUP should have helped to tackle this. 
The duration of untreated illness reported in our study is in line with other studies. The median DUI was 36 months (SD: 68.6) for the whole study sample, and DUI varied widely, which is similar to results described earlier (Häfner and Maurer, 2006; Riecher-Rössler et al., 2006).

Longer DUP and DUI were significantly associated with stronger negative symptoms. This is in line with most other studies as shown in the review by Marshall et al. (2005) which reported a significant association between DUP and several outcome measures including negative symptoms. In the meta analysis of Perkins et al. (REF), duration of initially untreated psychosis was even only associated with negative, but not with positive symptoms. In some studies finding a significant association between DUP and cognition, this finding ceased to be significant when it was controlled for negative symptoms (e.g. Amminger REF), suggesting that the influence of negative symptoms on cognition outruns the influence of DUP.

\subsection{DUP, DUI and cognitive impairment}

In this study, we could not detect significant relationships between DUP, DUI, and cognitive functioning, neither when cognitive functioning was looked at as raw outcome measure of neuropsychological tests, as a deterioration index, nor as the deviation of the performance of patients compared to the performance of healthy controls. As shown in the supplementary Table, this is in line with many other studies: among 18 studies examining this relationship, only 5 found a positive relationship.

The following theories could explain this:

There are several studies that suggest schizophrenic psychoses are neurodevelopmental disorders and that the origins of these are to be found in childhood (Jones et al., 1994). A recent 45-year follow-up report from a Copenhagen birth cohort showed that individuals who developed schizophrenic psychoses reached developmental milestones later than a control-group (Sorensen et al., 2010). These data confirm that the cognitive deficits in schizophrenic 
psychoses by far precede the onset of psychotic symptoms and therefore there might be no connection between the duration of untreated psychosis and cognitive performance.

Another important argument was proposed by Goldberg et al. (2009); the development of psychosis and neurocognitive deficits are based on two different psychological mechanisms: psychotic experiences could be the consequence of a learning process and are thus stabilized over time (Kapur, 2003). Hence they get the more difficult to treat, the longer they are being consolidated, which would explain the frequently reported correlation between DUP and treatment response (e.g. Perkins et al., 2005). In contrast to the development of psychotic experiences, moment-to-moment information processing is not learned. Therefore, DUP and cognition are not related to each other (Goldberg et al., 2009).

Using the approach of Amminger et al. (2002a) and applying the Deterioration Index (DI) by Wechsler (Wechsler, 1958), this study could not replicate the finding of a positive relationship between DUP and this index. This might be because we used a different test to calculate the DI than Amminger et al. (2002a). But as mentioned above; several studies suggest that schizophrenia is a neurodevelopmental disorder (e.g. Lewis and Levitt, 2002). Therefore, the concept of a premorbid IQ and a deterioration index seems inapplicable. It has additionally been criticized that the reliability of the $\mathrm{DI}$ is limited because the use of premorbid intellectual ability is problematic in cases of a long-standing learning problem (e.g. dyslexia) or in cases where damage to brain regions directly involved in those functions are present (e.g. Cipolotti and Warrington, 1995). In view of these arguments challenging the reliability of the DI, our results with it do not seem further surprising.

\subsection{Strengths/limitations}

The strengths of our study were that - in contrast to all other studies - we also included an ARMS sample and analysed how the DUI relates to their cognitive functioning. Additionally, we used different operationalizations for cognitive decline. 
A limitation is the modest sample size of this difficult-to-collect patient sample, which leads to limited statistical power. Furthermore, the used neuropsychological test versions imposed only a moderate cognitive load, which led to strong ceiling effects in some scores. This might have accounted for the lacking association between DUP, DUI and cognition. Additionally, we did not include a measure of memory, which has been shown to be an important marker within the area of at risk mental state samples (e.g. Lin et al / Valli et al).

\subsection{Conclusions}

This study could not - even when conceptualising cognition in different ways - confirm an association between duration of untreated psychosis or duration of untreated illness and neurocognition in FEP patients or ARMS individuals, suggesting that cognitive deficits occur prior to the onset of psychiatric symptoms (e.g. in childhood). However, as the relationship between DUP and treatment outcome is well established and as there might be other consequences of delayed treatment, this should not weaken the rationale for early detection and intervention strategies in patients with a first psychotic episode.

Nevertheless, the findings of the current study also suggest that in clinical practice, we should take care about clearly comprising patients amnestic cognitive development beginning in childhood and initiate cognitive training for those with early cognitive deficits if applicable. Future research investigating the relationship between DUP or DUI and cognition should therefore also encompass factors such as cognitive childhood development abnormalities. 


\section{References}

Allen, D.M., 1974. Relationship between Variable Selection and Data Augmentation and a Method for Prediction. Technometrics 16(1), 125-127.

Amminger, G.P., Edwards, J., Brewer, W.J., Harrigan, S., McGorry, P.D., 2002a. Duration of untreated psychosis and cognitive deterioration in first-episode schizophrenia. Schizophrenia Research 54(3), 223-230.

Amminger, G.P., Edwards, J., McGorry, P.D., 2002b. Estimating cognitive deterioration in schizophrenia. British Journal of Psychiatry 181, 164-164.

Andreasen, N.C., 1983. Scale for the assessment of negative symptoms. University of lowa, lowa City

Barnes, T.R.E., Hutton, S.B., Chapman, M.J., Mutsatsa, S., Puri, B.K., Joyce, E.M., 2000. West London first-episode study of schizophrenia - Clinical correlates of duration of untreated psychosis. British Journal of Psychiatry 177, 207-211.

Bilder, R.M., Lipschutz-Broch, L., Reiter, G., Geisler, S.H., Mayerhoff, D.I., Lieberman, J.A., 1992. Intellectual deficits in first-episode schizophrenia: evidence for progressive deterioration. Schizophrenia Bulletin 18(3), 437-448.

Bilder, R.M., Mukherjee, S., Rieder, R.O., Pandurangi, A.K., 1985. Symptomatic and Neuropsychological Components of Defect States. Schizophrenia Bulletin 11(3), 409-419.

Box, G.E.P., Cox, D.R., 1964. An analysis of transformations. Journal of the Royal Statistical Society: Series B 26(2), 211-252.

Cipolotti, L., Warrington, E.K., 1995. Neuropsychological assessment. Journal of Neurology, Neurosurgery \& Psychiatry 58(6), 655-664.

Drühe-Wienholt, C.M., Wienholt, W., 1998. CKV: Computergestütztes Kartensortierverfahren. Swets \& Zeitlinger B.V., Frankfurt am Main.

Gaynor, K., Dooley, B., Lawlor, E., Lawoyin, L.R., O'Callaghan, E., 2009. Cognitive deterioration and duration of untreated psychosis. Early Intervention in Psychiatry 3(2), 157-160.

Gediga, G., Schöttke, H., 1994. Turm von Hanoi - TvH, in: Hänsgen, K.-D. (Ed.). Hogrefe Testsystem (HTS), Göttingen.

Goldberg, T.E., Burdick, K.E., McCormack, J., Napolitano, B., Patel, R.C., Sevy, S.M., Goldman, R., Lencz, T., Malhotra, A.K., Kane, J.M., Robinson, D.G., 2009. Lack of an inverse relationship between duration of untreated psychosis and cognitive function in first episode schizophrenia. Schizophrenia Research 107(2-3), 262-266.

Häfner, H., Maurer, K., 2006. Early detection of schizophrenia: current evidence and future perspectives. World Psychiatry 5(3), 130-138.

Han, C., Kronmal, R., 2004. Box-Cox transformation of left-censored data with application to the analysis of coronary artery calcification and pharmacokinetic data. Statistics in Medicine 23(23), 3671-3679.

Heaton, R.K., Chelune, G.J., Talley, J.L., Kay, G.G., Curtis, G., 1993. Wisconsin Card Sorting Test Manual. Psychological Assessment Resources, Odessa (FL).

Horn, W., 1983. Leistungsprüfsystem L-P-S, Handanweisung. Hogrefe, Göttingen.

Jones, P., Murray, R., Rodgers, B., Marmot, M., 1994. Child developmental risk factors for adult schizophrenia in the British 1946 birth cohort. The Lancet 344(8934), 1398-1402.

Kapur, S., 2003. Psychosis as a state of aberrant salience: A framework linking biology, phenomenology, and pharmacology in schizophrenia. American Journal of Psychiatry 160(1), 13-23.

Lehrl, S., 1991. Manual zum MWT. Perimed-spitta, Balingen.

Lehrl, S., Triebig, G., Fischer, B., 1995. Multiple choice vocabulary test MWT as a valid and short test to estimate premorbid intelligence. Acta Neurologica Scandinavica 91(5), 335-345.

Lewis, D.A., Levitt, P., 2002. Schizophrenia as a disorder of neurodevelopment. Annual Review of Neuroscience 25, 409-432.

Marshall, M., Lewis, S., Lockwood, A., Drake, R., Jones, P., Croudace, T., 2005. Association between duration of untreated psychosis and in cohorts of first-episode outcome patients - A systematic review. Archives of General Psychiatry 62(9), 975-983.

Norman, R.M.G., Malla, A.K., 2001. Duration of untreated psychosis: a critical examination of the concept and its importance. Psychological Medicine 31(3), 381-400. 
Perkins, D.O., Gu, H.B., Boteva, K., Lieberman, J.A., 2005. Relationship between duration of untreated psychosis and outcome in first-episode schizophrenia: A critical review and meta-analysis. American Journal of Psychiatry 162(10), 1785-1804.

Pflueger, M.O., Gschwandtner, U., Stieglitz, R.D., Riecher-Rossler, A., 2007. Neuropsychological deficits in individuals with an at risk mental state for psychosis - Working memory as a potential trait marker. Schizophrenia Research 97(1-3), 14-24.

Riecher-Rossler, A., Aston, J., Ventura, J., Merlo, M., Borgwardt, S., Gschwandtner, U., Stieglitz, R.D., 2008. The Basel screening instrument for psychosis (BSIP): Development, structure, reliability and validity. Fortschritte Der Neurologie Psychiatrie 76(4), 207-216.

Riecher-Rössler, A., Aston, J., Ventura, J., Merlo, M., Borgwardt, S., Gschwandtner, U., Stieglitz, R.D., 2008. The Basel screening instrument for psychosis (BSIP): development, structure, reliability and validity. Fortschritte Neurologie und Psychiatrie 76(4), 207-216.

Riecher-Rössler, A., Gschwandtner, U., Aston, J., Borgwardt, S., Drewe, M., Fuhr, P., Pflueger, M., Radue, W., Schindler, C., Stieglitz, R.D., 2007a. The Basel early-detection-of-psychosis (FEPSY)study - design and preliminary results. Acta Psychiatrica Scandinavia 115(2), 114-125.

Riecher-Rössler, A., Gschwandtner, U., Aston, J., Borgwardt, S., Drewe, M., Fuhr, P., Pflüger, M., Radu, W., Schindler, C., Stieglitz, R.D., 2007b. The Basel early-detection-of-psychosis (FEPSY)-study design and preliminary results. Acta Psychiatrica Scandinavica 115(2), 114-125.

Riecher-Rössler, A., Gschwandtner, U., Borgwardt, S., Aston, J., Pflueger, M., Rössler, W., 2006. Early detection and treatment of schizophrenia: how early? Acta Psychiatrica Scandinavica 113, 73-80.

Riecher-Rössler, A., Pflueger, M.O., Aston, J., Borgwardt, S.J., Brewer, W.J., Gschwandtner, U., Stieglitz, R.D., 2009. Efficacy of using cognitive status in predicting psychosis: a 7-year follow-up. Biological Psychiatry 66(11), 1023-1030.

Rosvold, H.E., Mirsky, A.F., Sarason, I., Bransome, E.D., Beck, L.H., 1956. A Continuous PerformanceTest of Brain-Damage. Journal of Consulting Psychology 20(5), 343-350.

Sorensen, H.J., Mortensen, E.L., Schiffman, J., Reinisch, J.M., Maeda, J., Mednick, S.A., 2010. Early developmental milestones and risk of schizophrenia: A 45-year follow-up of the Copenhagen Perinatal Cohort. Schizophrenia Research 118(1-3), 41-47.

Ventura, J., Lukoff, D., Nuechterlein, K., Liberman, R., Green, M., Shaner, A., 1993. Manual of the expanded brief psychiatric rating scale. The International Journal of Methods in Psychiatric Research(3), 227-243.

Wechsler, D., 1958. The Measurement and Appraisal of Adult Intelligence. Williams and Wilkins Company, Baltimore.

Wechsler, D., 1981. Wechsler Adult Intelligence Scale-Revised (WAIS-R). The Psychological Corporation, New York.

Wyatt, R.J., 1991. Neuroleptics and the Natural Course of Schizophrenia. Schizophrenia Bulletin 17(2), 325-351.

Yung, A.R., Phillips, L.J., McGorry, P.D., McFarlane, C.A., Francey, S., Harrigan, S., Patton, G.C., Jackson, H.J., 1998. Prediction of psychosis - A step towards indicated prevention of schizophrenia. British Journal of Psychiatry 172, 14-20.

Zimmermann, P., Fimm, B., 1993. Testbatterie zur Aufmerksamkeitsprüfung (TAP) Version 1.02. Handbuch Teilo 1/2, Psytest, Freiburg. 
Table 1: Socio-demographic characteristics ${ }^{\S}$ of ARMS-T and FEP

\begin{tabular}{|c|c|c|c|c|c|}
\hline & \multicolumn{2}{|c|}{ ARMS-T* incl. $(n=24)$} & \multicolumn{2}{|c|}{ ARMS- $T^{* *}$ exclud. $(n=3)$} & \multirow{2}{*}{$\begin{array}{c}\text { Significance value } \\
\qquad p=0.54\end{array}$} \\
\hline Men & $\mathrm{n}=16$ & $(66.7 \%)$ & $\mathrm{n}=1$ & $(33.3 \%)$ & \\
\hline Women & $\mathrm{n}=8$ & $(33.3 \%)$ & $\mathrm{n}=2$ & $(66.7 \%)$ & \\
\hline Age & 26.6 & (6.9) & 21.0 & (3.1) & $p=0.06$ \\
\hline Education (in years) & 11.1 & (2.5) & 9.3 & $(1.2)$ & $p=0.10$ \\
\hline BPRS total & 41.0 & $(9.8)$ & 50.6 & $(35.6)$ & $p=0.69$ \\
\hline $\begin{array}{l}\text { BPRS positive } \\
\text { symptoms }\end{array}$ & 7.5 & $(2.2)$ & 9.3 & (6.7) & $p=0.67$ \\
\hline SANS total & 28.3 & $(18.1)$ & 17.3 & (6.9) & $p=0.09$ \\
\hline \multirow[t]{2}{*}{$\begin{array}{l}\text { Neuroleptic } \\
\text { medication }\end{array}$} & 3 & $(12.5 \%)$ & 0 & $(0.0 \%)$ & $p=1.00$ \\
\hline & \multicolumn{2}{|c|}{ FEP* includ. $(n=60)$} & \multicolumn{2}{|c|}{ FEP ${ }^{* \star}$ exclud. $(n=25)$} & Significance value \\
\hline Men & $\mathrm{n}=40$ & $(66.7 \%)$ & $n=14$ & $(56.0 \%)$ & $p=0.49$ \\
\hline Women & $n=20$ & $(33.3 \%)$ & $\mathrm{n}=11$ & $(44.0 \%)$ & \\
\hline Age & 30.1 & (8.2) & 29.3 & (9.4) & $p=0.72$ \\
\hline Education (in years) & 11.4 & (3.2) & 10.4 & $(2.6)$ & $p=0.14$ \\
\hline BPRS total & 52.2 & $(13.4)$ & 51.3 & (13.3) & $p=0.80$ \\
\hline $\begin{array}{l}\text { BPRS positive } \\
\text { symptoms }\end{array}$ & 12.4 & (3.6) & 11.4 & $(4.2)$ & $p=0.33$ \\
\hline SANS total & 26.5 & $(17.6)$ & 22.0 & $(19.4)$ & $p=0.37$ \\
\hline $\begin{array}{l}\text { Neuroleptic } \\
\text { medication }\end{array}$ & 23 & (38.3\%) & 6 & (35.3\%) & $p=0.96$ \\
\hline
\end{tabular}

ARMS-T, at risk mental state with transition to psychosis; FEP, first episode of psychosis

$\S$ Unless indicated otherwise values are given as means with SD in brackets

* With DUP/DUI and neuropsychological information

**Without DUP/DUI and/or neuropsychological information

${ }^{*}$ Fisher's exact test 
Table 2: Spearman rank correlations for duration of untreated psychosis (DUP) and duration of untreated illness (DUI) and normed composite scores/composite scores based on ztransformed scores of neuropsychological tests for the whole sample

\begin{tabular}{lllll}
\hline & $\mathrm{DUI}^{₹}$ & $\mathrm{DUl}^{\beta}$ & $\mathrm{DUP}^{¥}$ & DUP $^{\beta}$ \\
\hline MWT-A & -0.04 & -0.04 & -0.04 & -0.04 \\
LPS & 0.15 & 0.15 & 0.23 & 0.23 \\
ToH & 0.01 & 0.00 & -0.05 & -0.06 \\
WCST & -0.09 & -0.12 & -0.18 & -0.14 \\
TAP go/no go & 0.04 & 0.01 & 0.07 & 0.06 \\
TAP wm & 0.07 & 0.08 & 0.19 & 0.16 \\
CPT-OX & -0.11 & 0.10 & -0.12 & 0.13 \\
\hline
\end{tabular}

MWT-A = Mehrfachwahl-Wortschatz; LPS = Leistungsprüfsystem, scale 3; ToH = Tower of Hanoi; WCST $=$ Wisconsin Card Sorting System; TAP go/no go $=$ Go/No-Go subtest of the Tests for Attentional

Performance; TAP wm $=$ Working Memory subtest of the TAP; CPT-OX $=$ Continuous Performance Test $* p<0.05$

$¥$ Correlations with normed composite scores

${ }^{\beta}$ Correlations with composite scores based on z-transformed scores 
Table 3: Spearman rank correlations duration of untreated psychosis (DUP) and duration of untreated illness (DUI) and normed composite scores/composite scores based on ztransformed scores of neuropsychological tests separately by ARMS-T and FEP patients

\begin{tabular}{|c|c|c|c|c|c|c|}
\hline & ARMS & & FEP & & & \\
\hline & DUI & $\mathrm{DUI} \mathrm{I}^{\beta}$ & DUI $^{*}$ & $\mathrm{DUI} \mathrm{I}^{\beta}$ & DUP & DUP ${ }^{\beta}$ \\
\hline MWT-A & 0.25 & 0.25 & -0.12 & -0.12 & -0.04 & -0.04 \\
\hline LPS & 0.22 & 0.22 & 0.09 & 0.09 & 0.23 & 0.23 \\
\hline $\mathrm{ToH}$ & 0.30 & 0.33 & -0.12 & -0.15 & -0.05 & -0.06 \\
\hline WCST & 0.30 & 0.18 & -0.22 & -0.22 & -0.18 & -0.14 \\
\hline TAP go/no go & 0.03 & -0.01 & 0.05 & 0.01 & 0.07 & 0.06 \\
\hline TAP wm & 0.00 & 0.06 & 0.10 & 0.09 & 0.19 & 0.16 \\
\hline CPT-OX & 0.05 & 0.00 & -0.17 & 0.14 & -0.12 & 0.13 \\
\hline
\end{tabular}

ARMS-T, at risk mental state with transition to psychosis; FEP, first episode of psychosis MWT-A = Mehrfachwahl-Wortschatz; LPS = Leistungsprüfsystem, scale 3; ToH = Tower of Hanoi; WCST $=$ Wisconsin Card Sorting System; TAP go/no go $=$ Go/No-Go subtest of the Tests for Attentional Performance; TAP wm $=$ Working Memory subtest of the TAP; CPT-OX = Continuous Performance Test ${ }^{*} p<0.05$

¥ Correlations with normed composite scores

${ }^{\beta}$ Correlations with composite scores based on z-transformed scores 


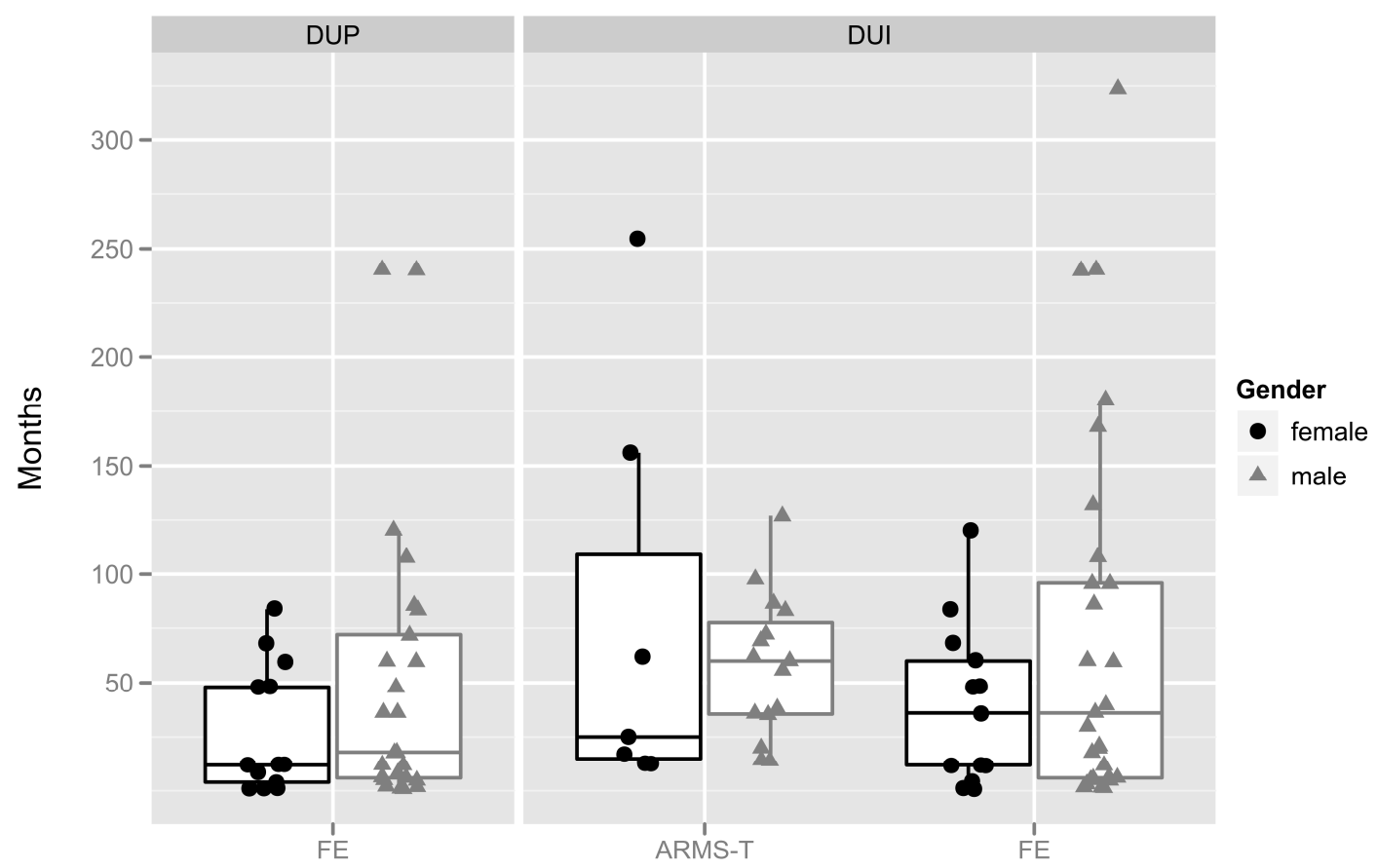

Figure 1: Duration of untreated illness (DUI) and duration of untreated psychosis (DUP) 


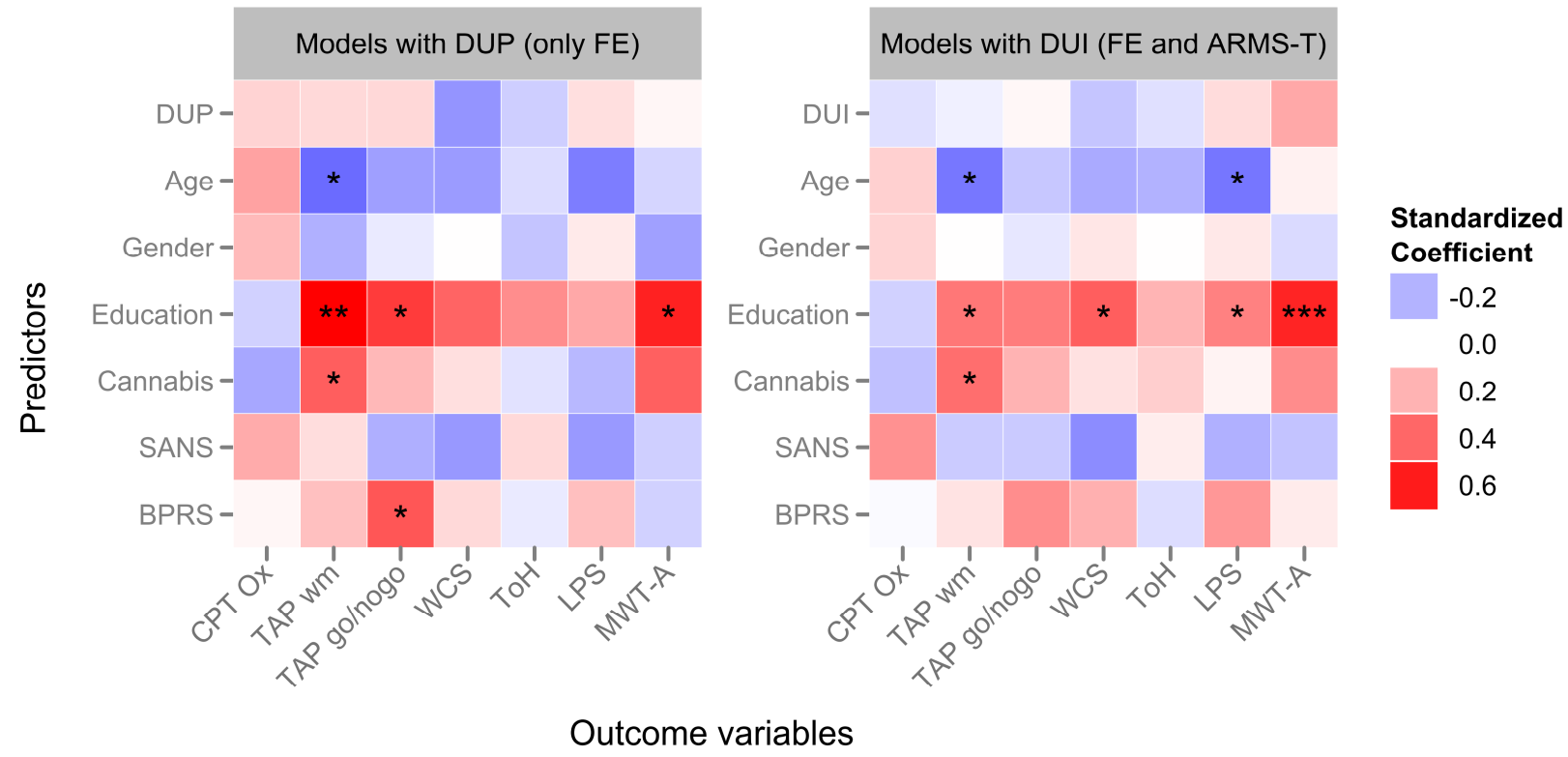

Figure 2: Predictors of cognition: cognitive test scores as dependent variables and log DUP/log DUI, age, gender, education, cannabis consumption, SANS negative and BPRS positive symptoms as independent variables

${ }^{*} \mathrm{p}<0.05 ;{ }^{* \star} \mathrm{p}<0.01 ;{ }^{* * *} \mathrm{p}<0.001$ after adjustment for multiple testing 